\title{
Decomposition of regular hypergraphs
}

\author{
Jeong-Ok Choi*and Douglas B. West ${ }^{\dagger}$ \\ Revised December 2015 and September 2016
}

\begin{abstract}
A $d$-block is a 0,1-matrix in which every row has sum $d$. Let $S_{n}$ be the set of pairs $(k, l)$ such that the columns of any $(k+l)$-block with $n$ rows split into a $k$-block and an $l$-block. For $n \geq 5$, we prove the general necessary condition that $(k, l) \in S_{n}$ only if each element of $\{1, \ldots, n\}$ divides $k$ or $l$. We also determine $S_{n}$ for $n \leq 5$. Trivially, $S_{1}=S_{2}=\mathbb{N} \times \mathbb{N}$. Also $S_{3}=\{(k, l): 2 \mid k l\}, S_{4}=\{(k, l): 6 \mid k l$ and $\min \{k, l\}>1\}$, and $S_{5}=\{(k, l): 3,4,5$ each divide $k$ or $l$, plus $11 \neq \min \{k, l\}>7\}$.
\end{abstract}

\section{Introduction}

Our problem is most simply expressed in the language of 0,1-matrices. A block is a 0,1 matrix $M$ whose rows all have the same sum; we denote the common sum by $\sigma(M)$. We use $d$-block to mean a block $M$ with $\sigma(M)=d$. Given a $(k+l)$-block with $k, l \in \mathbb{N}$ (where $\mathbb{N}$ is the set of positive integers), a $(k, l)$-split is a partition of the columns into two sets such that the resulting submatrices are a $k$-block and an $l$-block. A $d$-block $M$ is indecomposable if for all $(k, l)$ with $k+l=d$, there is no $(k, l)$-split of $M$.

Trivially, every $(k+l)$-block with one row has a $(k, l)$-split. This also holds for two rows, since columns of the forms $(0,1)^{T}$ and $(1,0)^{T}$ are equinumerous and can be paired. For $n \in \mathbb{N}$, let $S_{n}$ be the set of pairs $(k, l)$ such that every $(k+l)$-block with $n$ rows admits a $(k, l)$-split. Adding a row imposes additional restrictions, so $S_{n+1} \subseteq S_{n}$ for all $n$. We have noted $S_{1}=S_{2}=\mathbb{N} \times \mathbb{N}$. In this paper, we determine $S_{3}, S_{4}$, and $S_{5}$, and we prove a general necessary condition for $n \geq 5$.

Splitting of $d$-blocks into blocks with smaller row-sums has been studied in the language of hypergraphs. Each edge of a hypergraph is a subset of the vertex set, and distinct edges may have the same vertex set. A hypergraph is $d$-regular if every vertex lies in exactly $d$ edges. The incidence matrix of a hypergraph is the 0,1-matrix with rows indexed by

\footnotetext{
${ }^{*}$ Gwangju Institute of Science and Technology, South Korea, jchoi351@gist.ac.kr

†Zhejiang Normal University, Jinhua, China, and University of Illinois, Urbana IL, west@math.uiuc.edu. This research was partially supported by the National Security Agency under Award No. H98230-10-1-0363.
} 
the vertices and columns indexed by the edges such that entry $(v, e)$ is 1 if and only if vertex $v$ row belongs to edge $e$. Thus a hypergraph is $d$-regular if and only if its incidence matrix is a $d$-block. A regular hypergraph $H$ is indecomposable if has no nontrivial regular spanning proper subhypergraph, which is just the statement that its incidence matrix is an indecomposable $d$-block.

Motivated by questions in game theory (see the survey [4]), researchers studied the maximum possible degree of indecomposable regular hypergraphs with $n$ vertices. That is, the value $D(n)$ is the maximum $d$ such that some $d$-block with $n$ rows is indecomposable. Huckemann and Jurkat (see [4]) proved that $D(n)$ is finite for all $n$ (reproved in another way by Alon and Berman [1]), and with Shapley they proved $D(n) \leq(n+1)^{(n+1) / 2}$ (again see [4]). As a lower bound, Shapley proved $D(n)>2^{n-1} /(n-1)$ for $n>2$, improved to $D(n)>2^{n-3}$ for $n>2$ by van Lint and Pollak. Alon and Vũ [2] later proved the asymptotic formula $D(n)=n^{(1+o(1)) n / 2}$ (they showed in fact that this formula solves three problems). Their formula is close to the upper bound of Huckemann, Jurkat, and Shapley. Füredi [3] considered the restriction of the problem to hypergraphs in which every edge has size $t$. Kézdy, Lehel, and Powers [5] gave an application of the bounds on $D(n)$ to a problem involving weighted hypergraphs and the selection of a "consensus" vertex.

As far as we know, the exact values of $D(n)$ are known only for $1 \leq n \leq 5$ [4]; they are $1,1,2,3,5$, respectively. We will use these values to study $S_{n}$. Recall that $S_{n}=\mathbb{N} \times \mathbb{N}$ when $n \leq 2$. For $n=3$, a bit more thought yields $S_{3}=\{(k, l): 2 \mid k l\}$. We also prove $S_{4}=\{(k, l): 6 \mid k l$ and $\min \{k, l\}>1\}$ and $S_{5}=\{(k, l): 3,4,5$ each divide $k$ or $l$, and $11 \neq \min \{k, l\}>7\}$. Note that the condition for $S_{4}$ implies that $(1, l) \notin S_{n}$ for $l \in \mathbb{N}$ and $n \geq 4$. Thus there is no nontrivial $(d, n)$ such that every $d$-regular hypergraph with $n$ vertices has a perfect matching.

The divisibility requirement for $n=5$ is the special case for $n=5$ of a general necessary condition for membership in $S_{n}$, which we develop in Section 4:

Theorem 1.1. For $n \geq 5$, if $(k, l) \in S_{n}$, then each element of $\{1, \ldots, n\}$ divides $k$ or $l$.

For $2 \leq n \leq 4$, the condition is not quite necessary; changing $\{1, \ldots, n\}$ to $\{1, \ldots, n-1\}$ yields a weaker condition that characterizes $S_{n}$ in those cases. Since the result of van Lint and Pollak cited above yields $D(n)>n$ when $n \geq 6$, while $D(n)=n-1$ for $2 \leq n \leq 4$, we pose the following conjecture.

Conjecture 1.2. A necessary condition for $(k, l) \in S_{n}$ is that each element of $\{1, \ldots, D(n)\}$ divides $k$ or $l$. If $\min \{k, l\}$ is sufficiently large, then this condition is also sufficient.

Our results for $n \leq 5$ agree with Conjecture 1.2. Since $D(n) \geq n$ for $n \geq 6$, Conjecture 1.2 strengthens Theorem 1.1 .

We use the known values of $D(n)$ in proving both necessity and sufficiency of the description of $S_{n}$ for $n \leq 5$. Section 2 characterizes $S_{3}$ and outlines our general approach to 
determining $S_{n}$; the details for $n \in\{4,5\}$ follow in later sections. The method could perhaps settle Conjecture 1.2 for more values of $n$ once the corresponding values of $D(n)$ are known.

Although we use $D(n)$ to determine $S_{n}$, it is worth noting that $(k, l) \in S_{n}$ implies $D(n)<k+l$. Hence $m(n)>D(n)$, where $m(n)=\min \left\{k+l:(k, l) \in S_{n}\right\}$. A referee pointed out that, due to the divisibility requirement, Conjecture 1.2 would yield a much larger threshold: $m(n) \geq e^{D(n) / 2+o(D(n))}$.

\section{General approach}

To illustrate our method, we first characterize $S_{3}$. An equivalent statement was proved by André Kündgen (unpublished). When $A$ and $B$ are matrices with the same number of rows, let $A: B$ denote their concatenation, taking the union of the column sets as multisets. We use $m A$ to denote the concatenation of $m$ copies of $A$. Also, when $B$ is a submatrix of $A$ consisting of full columns, let $A \backslash B$ denote the matrix obtained by deleting those columns. We say that $B$ is a block in $M$ when $B$ is a block consisting of full columns of $M$.

Theorem 2.1. $S_{3}=\{(k, l): 2 \mid k l\}$

Proof. Let $M_{1}$ and $M_{2}$ be the 1-block and 2-block with three rows shown below. Note that $M_{2}$ is indecomposable; it contains no 1-block.

$$
M_{1}=\left[\begin{array}{l}
1 \\
1 \\
1
\end{array}\right], \quad M_{2}=\left[\begin{array}{lll}
0 & 1 & 1 \\
1 & 0 & 1 \\
1 & 1 & 0
\end{array}\right]
$$

Necessity. Let $M=\frac{k+l}{2} M_{2}$; this is a $(k+l)$-block. We claim that every block in $M$ has even row-sum. If a block in $M$ with odd row-sum contains a copy of each column of $M_{2}$, then deleting these three columns yields a smaller block with odd row-sum. Hence a minimal block $B$ among those with odd row-sum uses only copies of at most two columns of $M_{2}$. Now some row has no 0 , while another row does have a 0 , so $B$ is not a block. Thus if $k l$ is odd, then $M$ is a $(k+l)$-block with no $(k, l)$-split; this yields $(k, l) \notin S_{3}$.

Sufficiency. By symmetry, we may assume $2 \mid k$. Since $k \geq 2$ and $l \geq 1$, we have $d \geq 3$, where $d=k+l$. Since $D(3)=2$, every $d$-block $M$ with $d \geq 3$ (and three rows) decomposes into blocks with row-sum at most 2. We therefore obtain a 2-block $A$ in $M$ (possibly the concatenation of two 1-blocks). When $k=2$, this completes the decomposition. To complete a proof by induction on $k$, when $k>2$ we combine $A$ with a $(k-2)$-block from the $(k-2, l)$ split that the induction hypothesis guarantees for $M \backslash A$.

The sufficiency proof that the pairs not excluded from $S_{n}$ actually do belong to $S_{n}$ uses the value of $D(n)$ and induction on $k+l$. For the base case, we will need to check that when 
$(k, l)$ is in the specified set and $k+l \leq D(n)$, every $(k+l)$-block has a $(k, l)$-split. When $n \leq 5$, we have $D(n) \leq n$, and there is not much to check in the base case.

For the induction step, when $k+l>D(n)$, every $(k+l)$-block $M$ decomposes into blocks with row-sum at most $D(n)$. If we can always find a block $B$ in $M$ with $\sigma(B) \leq D(n)$ such that reducing $k$ or $l$ by $\sigma(B)$ yields another pair $\left(k^{\prime}, l^{\prime}\right)$ in the specified set, then combining $B$ with one of the blocks in a $\left(k^{\prime}, l^{\prime}\right)$-split of $M \backslash B$ guaranteed by the induction hypothesis completes the proof.

For the necessity of the characterization, our proofs that exclude a pair $(k, l)$ from $S_{n}$ are implementations of the next lemma, which we implicitly used in proving Theorem 2.1. Let $[m]$ denote the set $\{1, \ldots, m\}$, and let $M_{0}$ denote a matrix with no columns, so $M: M_{0}=M$.

Definition 2.2. A positive integer $q$ is n-robust if for all $r$ with $0 \leq r<q$, there exist an indecomposable $q$-block $M_{q}$ and an indecomposable $r$-block $M_{r}$ (both with $n$ rows) such that for all $p \in \mathbb{N}$ the row-sum of any block in $p M_{q}: M_{r}$ is congruent to 0 or $r$ modulo $q$.

Lemma 2.3. If $q$ is n-robust and $(k, l) \in S_{n}$, then $q$ divides $k$ or $l$.

Proof. Suppose that $q$ is $n$-robust and does not divide $k$ or $l$. Let $s=\lfloor k / q\rfloor$ and $i=k-s q$, and let $t=\lfloor l / q\rfloor$ and $j=l-t q$. Choose $r \in[q]$ such that $r \equiv i+j \bmod q$. Given the resulting $M_{q}$ and $M_{r}$ guaranteed by the definition of $n$-robust, let $M=(s+t) M_{q}: M_{r}$ or $M=(s+t+1) M_{q}: M_{r}$, depending on whether $i+j \leq q$ or not. Now $M$ is a $(k+l)$-block. By the definition of $n$-robust, $M$ does not contain a $k$-block, so $(k, l) \notin S_{n}$.

Note that if $q$ is $n$-robust, then $q \leq D(n)$; this motivates Conjecture 1.2. The difficulty in applying Lemma 2.3 is finding the needed $q$-block and $r$-block (for each $r$ ) and checking that the concatenations do not contain blocks with undesirable row-sums. The lemma does not save any work; it only states the plan. If we supply the specified indecomposable $q$-block and $r$-block for each prime power $q$ up to $D(n)$ and each $r \in[q]$, then a necessary condition for $(k, l) \in S_{n}$ will be that each prime power up to $D(n)$ divides $k$ or $l$.

It is not known whether there is an indecomposable $d$-block with $n$ rows (indecomposable $d$-regular $n$-vertex hypergraph) whenever $d<D(n)$. Nevertheless, our characterization of $S_{n}$ for $n \leq 5$ includes exhibiting such blocks for $n \leq 5$.

Recall that $S_{n+1} \subseteq S_{n}$. Since $(k, l) \in S_{3}$ requires $k l$ to be even, it therefore follows for $n \geq 3$ that $k l$ must be even when $(k, l) \in S_{n}$. For larger $n$ we can also eliminate the pairs containing a 1 .

Definition 2.4. Let $M_{1}, M_{2}$, and $M_{3}$ henceforth denote the matrices below.

$$
M_{1}=\left[\begin{array}{l}
1 \\
1 \\
1 \\
1
\end{array}\right] \quad M_{2}=\left[\begin{array}{lll}
0 & 1 & 1 \\
1 & 0 & 1 \\
1 & 1 & 0 \\
1 & 1 & 0
\end{array}\right] \quad M_{3}=\left[\begin{array}{llll}
0 & 1 & 1 & 1 \\
1 & 0 & 1 & 1 \\
1 & 1 & 0 & 1 \\
1 & 1 & 1 & 0
\end{array}\right]
$$


Lemma 2.5. If $n \geq 4$, then no pair $(k, l)$ with $k=1$ belongs to $S_{n}$.

Proof. Since $S_{n+1} \subseteq S_{n}$, it suffices to prove this for $n=4$. If $(k, l) \in S_{4}$, then $k l$ must be even. Thus it suffices to provide, for each even $l$, an $(l+1)$-block containing no 1-block. With $M_{1}, M_{2}, M_{3}$ defined as above, let $M=(l / 2-1) M_{2}: M_{3}$. Since $M_{2}$ is a 2-block and $M_{3}$ is a 3-block, $M$ is an $(l+1)$-block. Since no column of $M$ equals $M_{1}$ and the sum of any two columns has at least one $2, M$ contains no 1-block.

\section{Matrices with four rows}

When $n=4$, the matrices $M_{1}, M_{2}, M_{3}$ will play the roles of the matrices needed to apply the necessary condition in Lemma 2.3. When we speak of a matrix having a particular "form", we are allowing permutations of the columns.

Lemma 3.1. For $p \in \mathbb{N}$ and $0 \leq r<q \leq 3$, every block in $p M_{q}: M_{r}$ has the form $p^{\prime} M_{q}$ or $p^{\prime} M_{q}: M_{r}$ for some $p^{\prime}$. Consequently, if $(k, l) \in S_{n}$ for $n \geq 4$, then 3 divides $k$ or $l$.

Proof. The second statement follows from the first by Lemma 2.3 and $S_{n+1} \subseteq S_{n}$, since every block of the specified form has row-sum congruent to 0 or $r$ modulo $q$. For $q \leq 2$ the first statement is trivial, so assume $q=3$.

Let $B$ be a smallest block in $p M_{3}: M_{r}$ not having the desired form. If $B$ contains a copy of each column in $M_{3}$, then there is a smaller such block. Hence we may assume that some column of $M_{3}$ is not use in $B$.

For $r=0$, now $B$ has a row with no 0 and a row with 0 and is not a block.

For $r=1$, by the previous case we may assume that $B$ contains the column $M_{1}$. A block $B$ containing $M_{1}$ consists of that column and a (possibly empty) block from $p M_{3}$. Hence $B$ has the specified form.

For $r=2$, the block $B$ must use the one column in $M_{2}$ not in $M_{3}$. If $B$ contains copies of both other columns of $M_{2}$, then the case $r=0$ applies. Otherwise, $B$ has a row having no 0 and a row that has a 0 and cannot be a block.

Theorem 3.2. $(k, l) \in S_{4}$ if and only if $6 \mid k l$ and $\min \{k, l\} \neq 1$.

Proof. By Theorem 2.1 and $S_{n+1} \subseteq S_{n}$, we have $2 \mid k l$. By Lemma 3.1, we have $3 \mid k l$. By Lemma $2.5, \min \{k, l\} \neq 1$. Hence the conditions are necessary.

For sufficiency, suppose that $6 \mid k l$. By symmetry, there are two cases: either $k=3 s$ and $l=2 t$ for positive integers $s$ and $t$, or $k=6 s$ with $s$ a positive integer and $l \geq 2$.

Case 1. $k=3 s$ and $l=2 t$ for positive integers $s$ and $t$. We use induction on $s+t$. Let $M$ be a $(k+l)$-block. Since $D(4)=3$, every $d$-block with $d \geq 4$ decomposes into 1-blocks, 2-blocks, and 3-blocks. If these are all 1-blocks, then $M$ contains a $k$-block. 
Thus, we may assume that $M$ contains a 3 -block or a 2-block $A$. If $A$ is a 3 -block and $s=1$, or $A$ is a 2-block and $t=1$ (covering the base case $(3,2)$ ), then $A$ or its complement is the desired $k$-block. Otherwise, we apply the induction hypothesis to $M \backslash A$ and combine $A$ with a $(k-3)$-block or a $(l-2)$-block in $M \backslash A$ to obtain a $k$-block or an $l$-block in $M$.

Case 2. $k=6 s$ and $l \geq 2$. We use induction on $l$. The cases with $l \in\{2,3,4\}$ appear in Case 1 as $(3 \cdot 2 s, 2 \cdot 1),(3 \cdot 1,2 \cdot 3 s)$, and $(3 \cdot 2 s, 2 \cdot 2)$, respectively. For $l \geq 5$, since we may assume that any $(k+l)$-block contains a 2 -block or a 3 -block $A$, we can apply the induction hypothesis using $k$ and $l-2$ or $l-3$ to $M \backslash A$.

\section{A General Necessary Condition}

In this section we prove that when $n \geq 5$ and $1 \leq q \leq n$, membership of $(k, l)$ in $S_{n}$ requires that $q$ divides $k$ or $l$. This necessary condition is not sufficient. In the next section, we will exclude additional pairs when $n=5$ to obtain the complete description of $S_{5}$.

Definition 4.1. For $1 \leq i \leq n$, we define an $i$-block $M_{i}(n)$ with $n$ rows. For $n=5$ these are listed below; note that $M_{3}(5), M_{4}(5), M_{5}(5)$ have 4,6,7 columns, respectively.

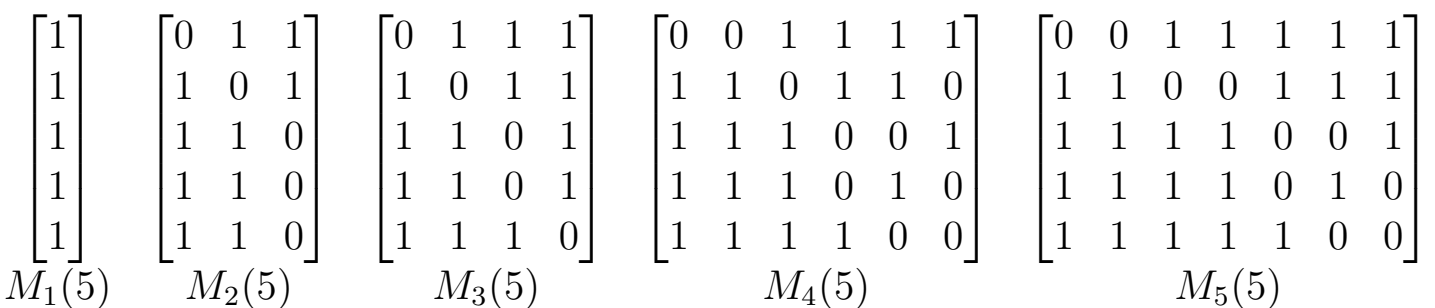

For $n \geq 6$, let $M_{1}(n)$ be the all-1 column vector, and define $M_{2}(n)$ by repeating the bottom row of $M_{2}(n-1)$. For $3 \leq q \leq n$, obtain $M_{q}(n)$ from $M_{q-1}(n-1)$ by first appending a 1 to the end of each row and then adding an $n$th row in which the first $q$ entries are 1 and the last one or two entries are 0 (one 0 when $q \leq n-2$, two when $q \in\{n-1, n\}$ ). Note that $M_{q}(n)$ has $q+1$ columns when $2 \leq q \leq n-2$ and $q+2$ columns When when $q \in\{n-1, n\}$. Graphically,

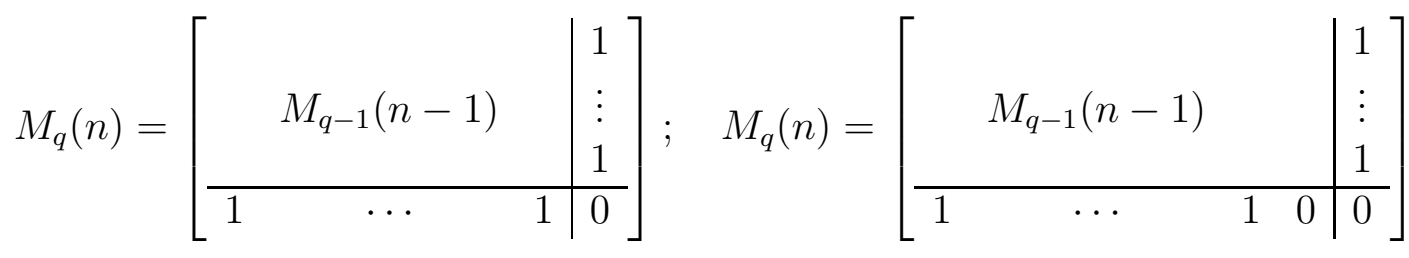

$$
\begin{aligned}
& \text { for } 3 \leq q \leq n-2 \text {; } \quad \text { for } n-1 \leq q \leq n \text {. }
\end{aligned}
$$

Note also that for $n=5$ the last column in $M_{q}(n)$ is unique and is not all 1 . Therefore, inductively we obtain the same statement for all $n \geq 5$; this property will be important. 
Lemma 4.2. For $n, p, q \in \mathbb{N}$ with $n \geq 5$ and $q \leq n$, every block in $p M_{q}(n)$ has the form $p^{\prime} M_{q}(n)$ for some $p^{\prime}$ with $p^{\prime} \leq p$.

Proof. The statement is trivial for $q=1$. For $q \in\{2,3\}$, the statement of the conclusion holds for the matrix $M_{q}$ of Definition 2.4, by Lemma 3.1. Hence it also holds for $p M_{q}(n)$, since the matrix $M_{q}(n)$ arises from $M_{q}$ by making extra copies of some row.

For $q \geq 4$, we use induction on $n$. We begin for $n=5$ by proving the statement for $q \in\{4,5\}$. First for $q=5$, let $B$ be a block in $p M_{5}(5)$. There are five types of columns from $M_{5}(5)$; from left to right, let $a, b, c, d$, e denote their multiplicities in $B$, respectively. Since a block must have the same number of $0 \mathrm{~s}$ in each row, the five row constraints give $a=b=c+d=c+e=d+e$. These equations require $a=b=2 c=2 d=2 e$, so there is just one parameter. Also, since $a=b=2 c$, we can view the block as having equal multiplicity for each of the seven columns of $M_{5}(5)$. Hence $B$ has the desired form.

For $q=4$ and $n=5$, let $B$ be a block in $p M_{4}(5)$. Each column of $M_{4}(5)$ appears in $M_{5}(5)$ except the last. Let $z$ be the multiplicity in $B$ of the last column of $M_{4}(5)$, and let $a, b, c, d$ be the multiplicities of the other columns of $M_{4}(5)$, named as in $M_{5}(5)$. Since each row of $B$ has the same number of 0 s, we obtain $a=b+z=c+d=c+z=d+z$. These equations require $a=2 b=2 c=2 d=2 z$, and we can view the block as having equal multiplicity for each of the six columns of $M_{4}(5)$. Hence $B$ has the desired form.

For the induction step, consider $n \geq 6$ and $4 \leq q \leq n$. Let $B$ be a block in $p M_{q}(n)$. Let $M^{\prime}$ be the matrix consisting of the first $n-1$ rows of $M_{q}(n)$. Let $v$ be the last column of $M_{q}(n)$, and let $z$ be the number of copies of $v$ in $B$. Let $B^{\prime}$ be the matrix obtained by removing the copies of $v$ from $B$ and deleting the last row. Since the copies of $v$ contributed $z$ to the sum of each row in $B$ other than the last row, $B^{\prime}$ is a block in $p M^{\prime}$.

Since $p M^{\prime}=p M_{q-1}(n-1)$, the induction hypothesis implies that $B^{\prime}$ consists of $p^{\prime}$ copies of each column of $M_{q-1}(n-1)$, for some $p^{\prime}$. Thus $\sigma\left(B^{\prime}\right)=p^{\prime}(q-1)$. Since $M_{q}(n-1)$ has $q$ copies of 1 in the bottom row before $v$, and each of those columns appears $p^{\prime}$ times in $B$, we have $\sigma(B)=p^{\prime} q$. Therefore, $z=p^{\prime}$, and $B$ has the desired form.

Setting $p=1$ in Lemma 4.2 yields the statement that $M_{q}(n)$ is indecomposable.

Lemma 4.3. For $p \in \mathbb{N}$, every block in $p M_{q}(5): M_{r}(5)$ for $0 \leq r<q \leq 5$ has the form $p^{\prime} M_{q}(5): M_{r}(5)$ or $p^{\prime} M_{q}(5)$ for some $p^{\prime}$ with $p^{\prime} \leq p$. In particular, $q$ is 5 -robust for $1 \leq q \leq 5$.

Proof. Lemma 4.2 is the case $r=0$. For $r=1$, since the one column of $M_{1}(5)$ is all $1 \mathrm{~s}$, Lemma 4.2 again applies.

Consider now $r>1$. Observe that every column of $M_{r}(5)$ lies in $M_{q}(5)$ except the last column of $M_{r}(5)$. Hence Lemma 4.2 implies that a block $B$ in $p M_{q}(5): M_{r}(5)$ with $\sigma(B)$ not divisible by $q$ must use the one copy of the last column of $M_{r}(5)$. Let the multiplicities of the other columns again be $a, b, c, d, e$, using the notation for columns of $M_{5}(5)$ as in Lemma 4.2. In considering $r \in\{2,3,4\}$, let $x, y, z$ respectively be the multiplicity of the last column in 
$M_{r}(5)$. Except for copies of that column, we count all columns used as copies of columns of $M_{q}(5)$.

We again count the 0 s in each row. For each case $(q, r)$, these counts appear below from row 1 to row 5 under "constraints from 0s"; the five values must be equal. The equalities allow us to compute all multiplicities in terms of $c$ as in the next section of the table. The final column then counts the 1s in each row of $B$. In each case, the row-sum is congruent to $r$ modulo $q$, and $B$ has the desired form.

\begin{tabular}{c|c|ccccc|cccccccc|c}
\hline$q$ & $r$ & \multicolumn{5}{|c|}{ constraints from 0s } & $a$ & $b$ & $c$ & $d$ & $e$ & $x$ & $y$ & $z$ & $\sigma(B)$ \\
\hline 3 & 2 & $a$ & $b$ & $c+1$ & $c+1$ & $y+1$ & $c+1$ & $c+1$ & $c$ & 0 & 0 & 1 & $c$ & 0 & $3 c+2$ \\
4 & 2 & $a$ & $b+z$ & $c+d+1$ & $c+z+1$ & $d+z+1$ & $2 c+1$ & $c+1$ & $c$ & $c$ & 0 & 1 & 0 & $c$ & $4 c+2$ \\
4 & 3 & $a$ & $b+z$ & $c+d$ & $c+z$ & $d+z+1$ & $2 c-1$ & $c$ & $c$ & $c-1$ & 0 & 0 & 1 & $c-1$ & $4 c-1$ \\
5 & 2 & $a$ & $b$ & $c+d+1$ & $c+e+1$ & $d+e+1$ & $2 c+1$ & $2 c+1$ & $c$ & $c$ & $c$ & 1 & 0 & 0 & $5 c+2$ \\
5 & 3 & $a$ & $b$ & $c+d$ & $c+e$ & $d+e+1$ & $2 c-1$ & $2 c-1$ & $c$ & $c-1$ & $c-1$ & 0 & 1 & 0 & $5 c-2$ \\
5 & 4 & $a$ & $b+1$ & $c+d$ & $c+e+1$ & $d+e+1$ & $2 c$ & $2 c-1$ & $c$ & $c$ & $c-1$ & 0 & 0 & 1 & $5 c-1$ \\
\hline
\end{tabular}

Lemma 4.4. For $p, n \in \mathbb{N}$ with $n \geq 5$, every block in $p M_{q}(5): M_{r}(5)$ for $0 \leq r<q \leq n$ has the form $p^{\prime} M_{q}(n): M_{r}(n)$ or $p^{\prime} M_{q}(n)$ for some $p^{\prime}$ with $p^{\prime} \leq p$. Thus $q$ is $n$-robust for $q \leq n$.

Proof. Lemma 4.3 is the case $n=5$; we use that as the basis for induction on $n$. For larger $n$, the claim for $r \leq 1$ is Lemma 4.2. Consider $r \geq 2$ (and hence $q>2$ ), and let $B$ be a block in $p M_{q}(n): M_{r}(n)$, with $t=\sigma(B)$.

Let $r^{\prime}=\max \{r-1,2\}$. Arrange the columns of $B$ by placing the copies of $\left[\begin{array}{lll}1 & \cdots & 0\end{array}\right]^{T}$ at the right end. This yields the following form of $B$.

$$
B=\left[\begin{array}{c|ccc}
M & & \multicolumn{2}{|c}{J} \\
& & & \\
\hline H & 0 & \cdots & 0
\end{array}\right],
$$

where $J$ is an all-1 matrix. Let $z$ be the number of columns in $J$. Since the last columns of $M_{q}(n)$ and $M_{r}(n)$ are unique, $M$ is a block in $p M_{q-1}(n-1): M_{r^{\prime}}(n-1)$, with $\sigma(M)=t-z$.

By the induction hypothesis, $M$ consists of the columns of $p^{\prime} M_{q-1}(n-1): M_{r^{\prime}}(n-1)$ or $p^{\prime} M_{q-1}(n-1)$, for some $p^{\prime}$ with $0 \leq p^{\prime} \leq p$. Thus $t-z$ is $p^{\prime}(q-1)+r^{\prime}$ or $p^{\prime}(q-1)$, respectively.

By the uniqueness of the final columns in $M_{q}(n)$ and $M_{r}(n)$, each copy of $M_{q-1}(n-1)$ or $M_{r^{\prime}}(n-1)$ in $M$ extends by adding the portion of $H$ below it to become the matrix obtained from $M_{q}(n)$ or $M_{r}(n)$ by deleting the final column (or that full matrix in the case of $r=2$ ). Thus $t \in\left\{p^{\prime} q+r, p^{\prime} q\right\}$, depending on whether $M_{r^{\prime}}(n-1)$ appears in $M$. We conclude that $z$ is $p^{\prime}+r-r^{\prime}$ or $p^{\prime}$, respectively. Hence the final columns provide exactly what is needed to conclude that $B$ has the form $p^{\prime} M_{q}(n): M_{r}(n)$ or $p^{\prime} M_{q}(n)$. 
Applying Lemma 2.3, we now have the following theorem.

Theorem 4.5. For $n \geq 5$, if $(k, l) \in S_{n}$, then $q \in\{1, \ldots, n\}$ divides $k$ or $l$.

\section{Matrices with five rows}

In this section, we determine $S_{5}$. Lemma 4.3 showed that $q$ is 5 -robust for $1 \leq q \leq 5$. Thus Lemma 2.3 implies that for every $(k, l) \in S_{5}$, each of $\{3,4,5\}$ divides $k$ or $l$. Also we have forbidden $\min \{k, l\}=1$. These conditions are not sufficient; the characterization of $S_{5}$ excludes additional pairs when $\min \{k, l\}$ is small.

Lemma 5.1. If $(k, l) \in S_{5}$, then $3,4,5$ each divide $k$ or $l$, plus $11 \neq \min \{k, l\}>7$.

Proof. By Lemma 4.3, the divisibility condition is necessary. Restricting to $k \geq l$, in this proof we exclude pairs of the form

$$
\{(20 s, 1),(20 s, 2),(20 s, 3),(15 s, 4),(12 s, 5),(20 s, 6),(20 s, 7),(20 s, 11)\}
$$

for each positive integer $s$. Lemma 4.3 already excludes $(20 s, 2),(20 s, 7)$, and $(20 s, 11)$ when $3 \nmid s$, and Lemma 2.5 excludes $(20 s, 1)$, but the argument here for the other cases also handles these.

For each case of $(k, l)$, we list below a $(k+l)$-block that we will show has no $(k, l)$-split. The matrices $M_{3}, M_{4}, M_{5}$ are as in Definition 4.1. We set $M=(\alpha s-\beta) M_{i}: \gamma M_{j}$ to consider $k=i \alpha s$ and $l=\gamma j-\beta i$. We group the cases by the matrix $M_{j}$. When $5 \mid k$, we use $M_{5}$ as the main repeated block; in the one case where $5 \nmid k$ and $l=5$, we use $M_{4}$.

\begin{tabular}{|c|c|c||c|c|c|}
\hline$k$ & $l$ & $(k+l)$-block $M$ & $k$ & $l$ & $(k+l)$-block $M$ \\
\hline $20 s$ & 1 & $(4 s-3) M_{5}: 4 M_{4}$ & $20 s$ & 2 & $(4 s-2) M_{5}: 4 M_{3}$ \\
$20 s$ & 6 & $(4 s-2) M_{5}: 4 M_{4}$ & $20 s$ & 7 & $(4 s-1) M_{5}: 4 M_{3}$ \\
$20 s$ & 11 & $(4 s-1) M_{5}: 4 M_{4}$ & $15 s$ & 4 & $(3 s-1) M_{5}: 3 M_{3}$ \\
$20 s$ & 3 & $(4 s-1) M_{5}: 2 M_{4}$ & $12 s$ & 5 & $(3 s-1) M_{4}: 3 M_{3}$ \\
\hline
\end{tabular}

In Lemma 4.2, we showed that blocks formed using only columns from $p M_{q}$ have row-sum divisible by $q$. Also, for $i<q \leq 5$, each column of $M_{i}$ except the last appears in $M_{q}$, and we showed that a block using one copy of this special column plus columns from $M_{q}$ has row-sum congruent to $i$ modulo $q$. In each case $l$ is outside the achievable class.

Now up to $\gamma$ copies of the exceptional column are available to use in forming an $l$-block. We use the same technique as before to eliminate these cases; the fact that we only need to exclude row-sum $l$ itself instead of a full congruence class is crucial.

For $M_{4}$, the special column is $(1,0,1,0,0)^{T}$; for $M_{3}$, it is $(1,1,1,1,0)^{T}$. Consider a block $B$ in $M$ using $x$ copies of the special column. Again let $a, b, c, d, e$, respectively, denote the number of copies of the five columns in $M_{5}$ that are used in $B$. In the last case, let $z$ be the 
number of copies of the rightmost column of $M_{4}$. As before, we first obtain constraints on these multiplicities by ensuring that all rows have the same number of 0s. These determine the multiplicities in terms of $c$ and $x$, which in turn yields a formula for the row sum. We then argue that $l$ is not achievable. In the computation, several cases combine.

\begin{tabular}{|c|c|c|c|c|c|c|c|c|c|c|}
\hline$M$ & \multicolumn{4}{|c|}{ constraints from 0s } & $a$ & $b$ & $c$ & $d$ & $e$ & $\sigma(B)$ \\
\hline$p M_{5}: \gamma M_{4}$ & $a b+x$ & $c+d$ & $c+e+x$ & $d+e+x$ & $2 c$ & $2 c-x$ & $c$ & $c$ & $c-x$ & $5 c-x$ \\
\hline$p M_{5}: 4 M_{3}$ & $a$ & $c+d$ & $c+e$ & $d+e+x$ & $2 c-x$ & $2 c-x$ & $c$ & $c-x$ & $c-x$ & $5 c-2 x$ \\
\hline$p M_{4}: 3 M_{3}$ & $a b+z$ & $c+d$ & $c+z$ & $d+z+x$ & $2 c-x$ & $c$ & $c$ & $c-x$ & 0 & $4 c-x$ \\
\hline
\end{tabular}

To form an $l$-block in $p M_{5}: \gamma M_{j}$, we need $x \leq q$. In each case, the requirement that $d$ and $e$ are nonnegative yields $c \geq x$. Also the row-sum in the repeated block $M_{i}$ is the coefficient on $c$ in $\sigma(B)$. To achieve $\sigma(B)=l$, this fixes the congruence class of $x$ modulo $i$. Since $x \leq \gamma$ and $c \geq x$, in each case this produces too large a value of $l$.

For $5 \mid k$ and $l \equiv 1 \bmod 5$, an $l$-block in $p M_{5}: 4 M_{4}$ requires $x=4$, but then $l \geq 16$.

For $5 \mid k$ and $l \equiv 2 \bmod 5$, an $l$-block in $p M_{5}: 4 M_{3}$ requires $x=4$, but then $l \geq 12$.

For $5 \mid k$ and $l=3$, an $l$-block in $p M_{5}: 2 M_{4}$ requires $x=2$, but then $l \geq 8$.

For $5 \mid k$ and $l=4$, an $l$-block in $p M_{5}: 3 M_{3}$ requires $x=3$, but then $l \geq 9$.

For $4 \mid k$ and $l=5$, an $l$-block in $p N_{4}: 3 M_{3}$ requires $x=3$, but then $l \geq 9$.

The final contradictions in the proof of Theorem 5.1 show how delicate these exceptions are. Each case requires $l$ to be at least in the next higher congruence class modulo $i$. Indeed, after excluding these small values of $l$, the conditions are sufficient.

Theorem 5.2. $(k, l) \in S_{5}$ if and only if $3,4,5$ each divide $k$ or $l$, and also $11 \neq \min \{k, l\}>7$.

Proof. Necessity was established in Lemma 5.1. For sufficiency, we consider explicitly the pairs that have not been excluded. We may assume by symmetry that $k$ is divisible by at least two of $\{3,4,5\}$. Note that the pairs $(60 r, 8),(60 r, 9)$, and $(60 r, 10)$ have the form $(15 s, 4 t),(20 s, 3 t)$, or $(12 s, 5 t)$, respectively. Hence it suffices to show that for $s \geq 1$ the following pairs lie in $S_{5}$ :

$$
\begin{array}{ll}
\{(20 s, 3 t): t \geq 3\} & \{(15 s, 4 t): t \geq 2\} \\
\{(12 s, 5 t): t \geq 2\} & \{(60 s, t): t \geq 12\} .
\end{array}
$$

Since $D(5)=5$, every block $M$ with five rows decomposes into indecomposable blocks with row-sums at most 5 . These blocks provide a partition of the integer $\sigma(M)$. Our task is to show that every partition of $k+l$ whose parts are all at most 5 splits into portions summing to $k$ and to $l$ when $(k, l)$ lies in a family listed above. Let $b_{r}$ be the number of copies of $r$ in the partition. We may assume that no two parts sum to at most 5 , because it then suffices to consider the partition obtained by replacing them with one part equal to their sum. In particular, $b_{1}+b_{2}+b_{3} \leq 1$, except that $b_{3}>1$ is possible when $b_{1}=b_{2}=0$. 
For each family, we use induction on $s+t$. In each family, the claim trivially holds for the degenerate case $s=0$. We first verify splittability for the instances with $s \geq 1$ and smallest $t$. Subsequently, we may assume that $s \geq 1$ and that $t$ exceeds the smallest value, which allows us by the induction hypothesis to assume when $l=j t$ that there is no $j$-block. In the last family, the gap for $l=11$ forces us to consider $(60 s, t)$ separately for $t \in\{8,9,10\}$.

Case 1: $(20 s, 3 t)$ with $t \geq 3$, so $\sigma(M) \geq 29$. For $t=3$ and $s \geq 1$, suppose that $M$ contains no 20-block or 9-block. We have $b_{3} \leq 2$ and $4 b_{4}+5 b_{5} \leq 16$, since $b_{4} b_{5}=0$. Hence $\sigma(M) \leq 22$, a contradiction. For larger $t$, we may assume $b_{3}=0, b_{4} \leq 4$, and $b_{5} \leq 3$. Also $b_{1}+2 b_{2} \leq 2$. Hence $\sigma(M) \leq 33$, which leaves only the case $(20,12)$. This arises only when $\left(b_{1}, b_{4}, b_{5}\right)=(1,4,3)$, but then three 4 -blocks yields the split.

Case 2: $(15 s, 4 t)$ with $t \geq 2$, so $\sigma(M) \geq 23$. For $t=2$ and $s \geq 1$, suppose that $M$ contains no 15 -block or 8-block. We have $b_{4} \leq 1$ and $3 b_{3}+5 b_{5} \leq 12$, since $b_{3} b_{5}=0$. Hence $\sigma(M) \leq 16$, a contradiction. For larger $t$, we may assume $b_{4}=0, b_{3} \leq 4$, and $b_{5} \leq 2$. Hence $\sigma(M) \leq 22$, a contradiction.

Case 3: $(12 s, 5 t)$ with $t \geq 2$, so $\sigma(M) \geq 22$. For $t=2$ and $s \geq 1$, suppose that $M$ contains no 12-block or 10-block. We have $b_{5} \leq 1$ and $3 b_{3}+4 b_{4} \leq 11$. Hence $\sigma(M) \leq 16$, a

contradiction. For larger $t$, we may assume $b_{5}=0, b_{4} \leq 2$, and $b_{3} \leq 3$. Hence $\sigma(M) \leq 17$, a contradiction.

Case 4: $(60 s, l)$ with $l \geq 12$. Since $D(5)=5$, it suffices to obtain the split for $12 \leq l \leq 16$. For $l \in\{12,15\}$, apply Case 1 above. For $(60 s, 13)$, any single part in $\{1,3,4,5\}$ reduces the search to an earlier case; since $60 s+13$ is odd, the parts cannot all equal 2. Similarly, for $(60 s, 14)$ it suffices to have a part in $\{1,2,4,5\}$, and the parts cannot all equal 3.

\section{References}

[1] N. Alon and K. Berman, Regular hypergraphs, Gordon's lemma, Steinitz's lemma and invariant theory, J. Combin. Theory Ser. A 43 (1986), 91-97.

[2] N. Alon and V.H. Vũ, Anti-Hadamard matrices, coin weighing, threshold gates, and indecomposable hypergraphs. J. Combin. Theory Ser. A 79 (1997), no. 1, 133-160.

[3] Z. Füredi, Indecomposable regular graphs and hypergraphs. Special volume to mark the centennial of Julius Petersen's "Die Theorie der regulären Graphs", Part II, Discrete Math. 101 (1992), no. 1-3, 59-64.

[4] J. E. Graver, A survey of the maximum depth problem for indecomposable exact covers, in:Infinite and Finite Sets, (Keszthely, 1973), Proc. Colloq. math. Soc. J. Bolyai, 10 (NorthHolland, Amsterdam, 1975), 731-743.

[5] A. Kézdy, J. Lehel, and R.C. Powers, Heavy transversals and indecomposable hypergraphs. Combinatorica 23 (2003), no. 2, 303-310. 